\title{
Expression of Calnexin Reflects Paneth Cell Differentiation and Function
}

\author{
Nikolaus Gassler, Martina Schnölzer, Claudia Rohr, Burkhard Helmke, \\ Jürgen Kartenbeck, Sylvia Grünewald, Rico Laage, Armin Schneider, \\ Bettina Kränzlin, Alfred Bach, Herwart F. Otto, and Frank Autschbach \\ Department of Pathology (NG, CR, BH, HFO, FA), Heidelberg University, German Cancer Research Center (MS, JK), \\ and Axaron Bioscience AG (SG, RL, AS, AB), Heidelberg, and ZMF (BK), Heidelberg University, \\ Mannheim, Germany
}

\begin{abstract}
SUMMARY: It has been suggested that the behavior and function of Paneth cells in metaplasia are different from those found in normal intestinal mucosa. In this study, we investigated whether calnexin, a protein involved in secretory pathways, might be associated with differentiation and function of Paneth cells in normal small intestine, in complete intestinal metaplasia of the stomach, and in Paneth cell-rich adenomas. Differentiation and function of Paneth cells was monitored by Ki67, lysozyme, and morphologic features. Using a newly established monoclonal antibody, we found that calnexin is regularly synthesized by Paneth cells of normal small intestine. In these cells, the staining intensity of calnexin was inversely correlated with their content of secretory granules (lysozyme). In contrast, Paneth cells of intestinal metaplasia and Paneth cell-rich adenomas showed a reduced immunostaining of both calnexin and lysozyme. Moreover, these Paneth cells synthesized the proliferation marker Ki67, a phenomenon that was never observed in Paneth cells of normal small intestine. In vitro experiments using CaCo2 cells showed that the expression of calnexin is not directly affected by the induction of mitosis. In conclusion, calnexin probably reflects the status of Paneth cell differentiation and function. The results do not necessarily indicate that calnexin has a function in Paneth cell proliferation. (Lab Invest 2002, 82:1647-1659).
\end{abstract}

$T$ he four principal epithelial cell types of the human small intestinal mucosa (absorptive, goblet, Paneth, and endocrine cells) derive from a multipotent stem cell located near the base of crypts of Lieberkühn. In contrast to absorptive and goblet cells, which migrate in approximately 5 days from the crypt depths to the surface, endocrine cells and Paneth cells differentiate during a downward migration to the base of crypts. Paneth cells reside there for approximately 20 days (Bry et al, 1994). Paneth cells are generally recognized in hematoxylin and eosinstained sections by their large eosinophilic apical secretory granules and their characteristic ultrastructural pattern (Otto, 1973). Functions that have been attributed to Paneth cells include modulation of the intestinal microflora and maintenance of the mucosal defense barrier through production and secretion of several antimicrobial peptides, such as lysozyme, secretory phospholipase $\mathrm{A}_{2}$, and $\alpha$-defensins (Nevalainen et al, 1998; Ouellette, 1999; Ouellette and Bevins, 2001). In addition, Paneth cells can also produce further regulatory molecules including growth

DOI: 10.1097/01.LAB.0000041709.42598.4A

Received September 13, 2002.

The study was in part supported by the Deutsche Forschungsgemeinschaft (SFB 405) and the Tumorzentrum Heidelberg/Mannheim (I./1./2.).

Address reprint requests to: Dr. N. Gassler, Universität Heidelberg, Pathologisches Institut, INF 220/ 221, D-69120 Heidelberg, Germany. E-mail:Nikolaus_Gassler@med.uni-heidelberg.de factors, suggesting that Paneth cells may regulate the proliferation and differentiation programs of epithelial cell lineages by short-circuit paracrine loops (Bry et al, 1994; Ganz, 2000).

In humans, Paneth cell metaplasia is preferentially found in atrophic gastritis (Wong et al, 2000). Other nonmalignant pathologic conditions associated with Paneth cell metaplasia are diversion colitis, ulcerative colitis, and collagenous colitis (Goff et al, 1997; Shah et al, 1998; Stamp et al, 1992; Vujanic and Dojcinov, 2000; Weaver et al, 1992). Although the metaplastic tissue of the stomach has some morphologic features in common with the small intestinal mucosa, a series of studies has provided evidence that cellular differentiation in metaplastic tissues is different from that found in normal intestinal mucosa (Cahill et al, 1996; Inada et al, 2001; Wong et al, 2000; Yabuki et al, 1997). It is suggested that the normal growth control processes of Paneth cells are disturbed in complete intestinal metaplasia (Wong et al, 2000). Thus, the presence of intestinal metaplasia of the stomach is thought to increase the risk for developing gastric cancer (Filipe et al, 1994; Stemmermann, 1994; Wong et al, 2000; You et al, 1993). However, malignant transformation of intestinal Paneth cells into "Paneth cell-carcinoma" has not been established yet, but Paneth cells and/or Paneth-like cells are frequently found in malignancies of the stomach and the colon (Correa, 1988; Heitz and Wegmann, 1980; Pai et al, 1998; Wong et al, 2000). 
Calnexin (Cnx) is characterized as a membranebound nonclassical lectin-like molecule that binds transiently to virtually all newly synthesized glycoproteins (Bergeron et al, 1994; Helenius and Aebi, 2001). The luminal domain of $\mathrm{Cnx}$ contains a lectin domain, as well as a distinct loop structure that might be involved in direct protein-protein interactions (Chevet et al, 2001). The Cnx molecule interacts in a unique chaperone system found in the endoplasmic reticulum (ER) of nearly all eukaryotes, the so-called Cnxcalreticulin cycle (Parodi, 2000). Cnx and other ERassociated molecules allow incorrectly folded proteins to acquire their correct conformation. Foldingcompetent states of the new synthesized proteins are maintained by these molecules (Chevet et al, 2001). Therefore, it has been suggested that expression of Cnx is important for secretory activities of cells.

The aim of the study was to investigate whether the expression of $\mathrm{Cnx}$, a protein involved in secretory pathways, might be associated with cellular differentiation and cell-cycle activity of Paneth cells. For this purpose normal mucosa of human small intestine, Paneth cell metaplasia, Paneth cell-rich adenomas, and $\mathrm{CaCo} 2$ cells were investigated using a newly generated rat $\mathrm{mAb}$. We hypothesized that expression and distribution of $\mathrm{Cnx}$ reflects Paneth cell differentiation and function.

\section{Results}

\section{Characterization of the Anti-Cnx mAb}

The affinity-purified supernatant of the BB1F10 hybridoma cell line was used in all experiments. First, the $\mathrm{mAb}$ was used for immunoscreening experiments (Fig. 1, A and B). Two specifically recognized plaques were identified and further plaque purified. Full-length sequencing ( $\mathrm{Cnx}$ CDS 1778 bases) revealed human $\mathrm{Cnx}$ in both cases (accession number BC003552). Second, immunoaffinity chromatography was performed, and a single product of approximately $85 \mathrm{kDa}$ was prepared from the elutions by $7.5 \%$ SDS-PAGE (Fig. 1C). The isolated $85-\mathrm{kDa}$ protein was identified as human $\mathrm{Cnx}$ (BC003552) by matrix-assisted laser desorption/ionization (MALDI) mass spectrometry (Fig. 1D). The mAb was subsequently further characterized as IgG2a by isotype-specific mapping (Fig. 1E).

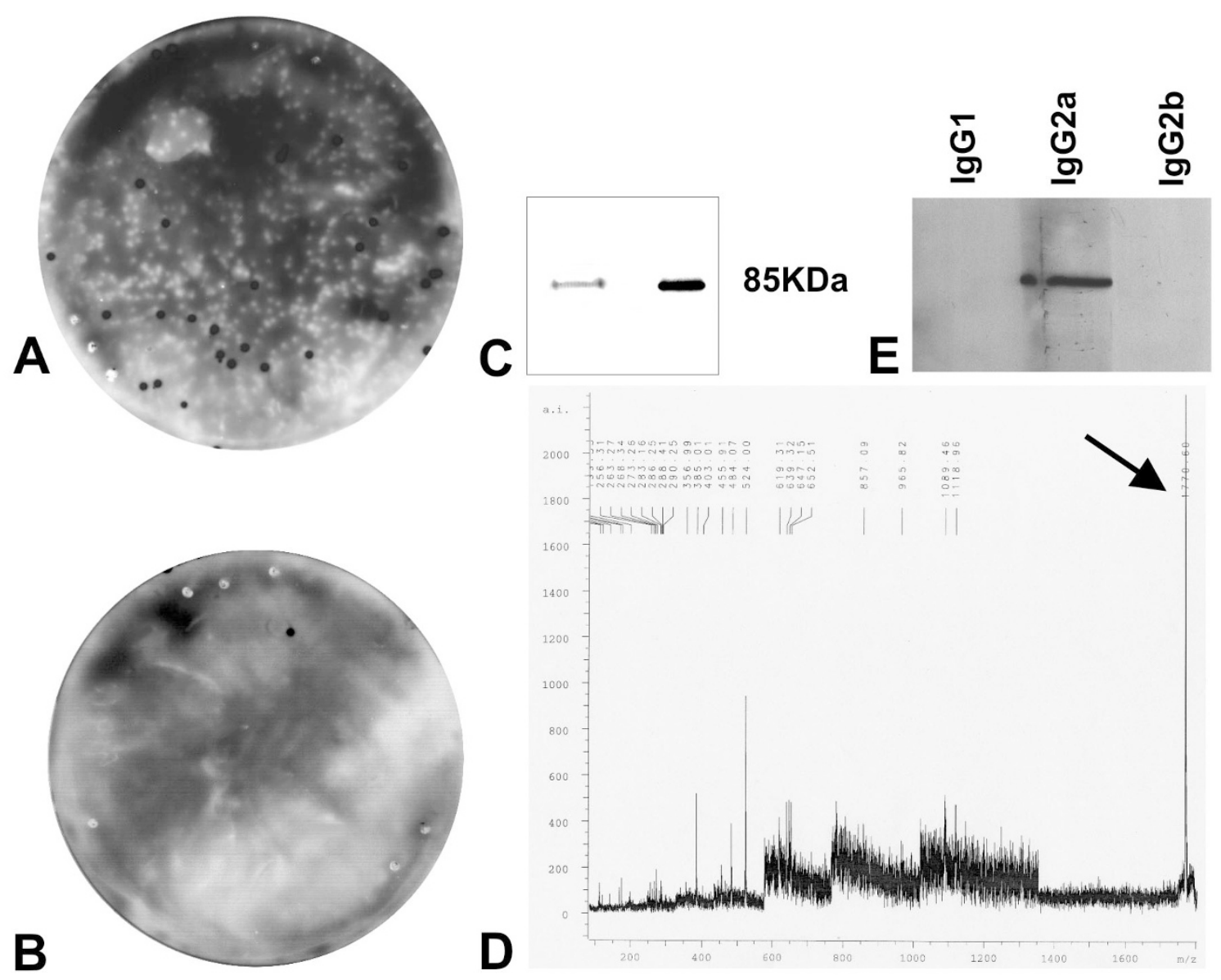

Figure 1.

Characterization of the anti-calnexin ( $\mathrm{Cnx}$ ) antibody. A and B, Immunoscreening experiments. A, Second step in plaque purification: clones expressing Cnx (black) and clones without any $\mathrm{Cnx}$ expression (white) exist together on a filter (ECL staining). B, Last step in plaque purification: one isolated clone expressing $\mathrm{Cnx}$ is shown (black; ECL staining). Note, white points in the periphery are used for filter orientation. C, One-dimensional SDS-PAGE of eluted proteins after immunoaffinity chromatography (left). The single reaction product of approximately $85 \mathrm{kDa}$ shows immunoreactivity against Cnx in Western blot analysis (right). D, The $85-\mathrm{kDa}$ protein isolated from the SDS-PAGE gel is identified as human $\mathrm{Cnx}$ by matrix-assisted laser desorption/ionization (MALDI)-PSD fragment ion analysis of the parent ion at $\mathrm{m} / \mathrm{z} 1770.60$ (arrow, BC003552). E, Isotype-specific mapping reveals that the mAb against $\mathrm{Cnx}$ is a rat IgG2a subtype. 


\section{Expression of Cnx in Paneth Cells of Normal Small Intestine}

In epithelial cells of normal small intestinal mucosa, a strong signal for $\mathrm{Cnx}$ was seen at villi and at the base of crypts (Fig. 2, A and B). In crypts, these epithelial cells were morphologically identified in serial sections as Paneth cells by their large apical eosinophilic secretory granules. Interestingly, strong immunostaining of the Cnx protein was not found in every Paneth cell (Fig. 2C). Cnx immunostaining was clearly diminished in Paneth cells containing a large amount of secretory granules. Occasionally, nuclear shrinkage/ karyorrhexis was additionally seen in such cells. Cnx was also found in endocrine cells and in stem cells (Fig. 2D). Endocrine cells were identified by the basal localization of secretory granules and immunohistochemical staining of chromogranin (data not shown), whereas stem cells were characterized by mitotic figures and nuclear immunostaining of the Ki67 protein. The synthesis of $\mathrm{Cnx}$ in normal small intestine and normal gastric mucosa (Fig. 2E) was corroborated by immunoblots (Fig. 2F). In normal small intestinal tissues, the distribution of $\mathrm{Cnx}, \mathrm{Ki} 67$, and the Paneth cell-metabolite lysozyme was further investigated by triple immunofluorescence (Fig. 3, A to F). Notably, strong immunostaining of lysozyme was found in those Paneth cells that did show a weak immunostaining of the Cnx protein (Fig. 3, A to C). Nuclear Ki67 protein was never seen in Paneth cells of normal small intestine (Fig. 3, D and E). Cnx was further evaluated by mRNA in situ hybridization experiments. Cnx mRNA were found in Paneth cells and in enterocytes of villi (Fig. 3, G to I). All appropriate negative controls yielded completely negative results. The data are summarized in Table 1.

\section{Expression of Cnx in Paneth Cell Metaplasia}

In complete intestinal metaplasia, strong immunostaining of the $\mathrm{Cnx}$ protein was found in only some Paneth cells (Fig. 4, A to C). In contrast to the unaffected small intestinal tissue, the diminished $\mathrm{Cnx}$ staining did not always correlate with the accumulation of secretory granules. In complete intestinal metaplasia, notably, the Ki67 protein was found in the nuclei of some Paneth cells (Fig. 4D). Such cells displayed a very low costaining for both $\mathrm{Cnx}$ and the Paneth cell marker lysozyme (Fig. 4E). These immunohistochemical data were corroborated by mRNA in situ hybridization experiments (Fig. 4, G to I; Table 1). In all cases, negative controls yielded completely negative results.

\section{Expression of Cnx in Paneth Cell-Rich Adenomas}

In all cases of Paneth cell-rich adenomas investigated $(n=5)$, detection of Cnx protein, Cnx mRNA, and lysozyme always revealed weak immunostaining when compared with $\mathrm{Cnx}$ and lysozyme in normal ileal tissues (Fig. 5, A-I). Moreover, strong nuclear immunofluorescence of the Ki67 protein was regularly found in these cells (Fig. 5, Table 1); negative controls yielded completely negative results.

\section{Expression of Cnx in CaCo2 Cells}

To investigate expression and distribution of $\mathrm{Cnx}$ during cellular proliferation (mitosis), additional cell culture experiments were performed. As previously described, cellular proliferation/mitosis of $\mathrm{CaCo} 2$ cells dramatically increased after treatment with thrombinactivated factor XIII (FXIII) (Cario et al, 1999). The distribution of the $\mathrm{Cnx}$ protein changed in mitosis (Fig. 6, A to F). In interphase $\mathrm{CaCo} 2$ cells, the $\mathrm{Cnx}$ protein was distributed in a fine reticulum filling the cytoplasm, concentrated near the nuclear membrane. In prophase and prometaphase, condensation of DNA and establishment of chromosomes were accompanied by condensation of the cytoplasm including the Cnx protein. Immunostaining of the Cnx protein was most prominent in metaphase and early anaphase. In late anaphase and telophase, the distribution of $\mathrm{Cnx}$ was increasingly reticular, ultimately concentrated near the newly established nuclear membrane. In every case, the mean area containing the chromosomes was lacking the Cnx protein, whereas small amounts of $\mathrm{Cnx}$ were regularly found in the area containing the spindle apparatus. Quantitative analysis of the Cnx protein expression by Western blot technique after 24, 48, and 60 hours of incubation revealed that the total expression of $\mathrm{Cnx}$ was unchanged in thrombin-activated FXIII-treated $\mathrm{CaCo} 2$ cells (high cellular proliferative activity) when compared with untreated $\mathrm{CaCo} 2$ cells (low cellular proliferative activity) (Fig. 7).

\section{Discussion}

Although a high number of molecules are secreted by Paneth cells (Bry et al, 1994; Ganz, 2000; Nevalainen et al, 1998; Ouellette, 1997, 1999; Ouellette and Bevins, 2001), there is no systematic analysis of the secretory/signaling pathways or receptors by which these cells regulate differentiation and function/secretion. Cnx, a multifunctional molecule, is generally involved in secretory pathways and interacts in a unique chaperone system (Bergeron et al, 1994; Helenius and Aebi, 2001; Parodi, 2000). In this study, the expression and synthesis of $\mathrm{Cnx}$ in Paneth cells was investigated.

In normal small intestine, Cnx was strongly expressed and synthesized by Paneth cells without storage of secretory granules in their cytoplasm. Immunostaining of the $\mathrm{Cnx}$ protein was diminished with advanced stage of cell life, indicated by an increased density of secretory granules (immunostaining of lysozyme). Moreover, the immunostaining of $\mathrm{Cnx}$ was further decreased in the case of nuclear shrinkage/ karyorrhexis (late stage of cell life). The lack of nuclear expression of Ki67 protein in normal Paneth cells is confirmed by previously recorded data, which show that these cells are postmitotic and derive from a multipotent stem cell located near the base of crypts 

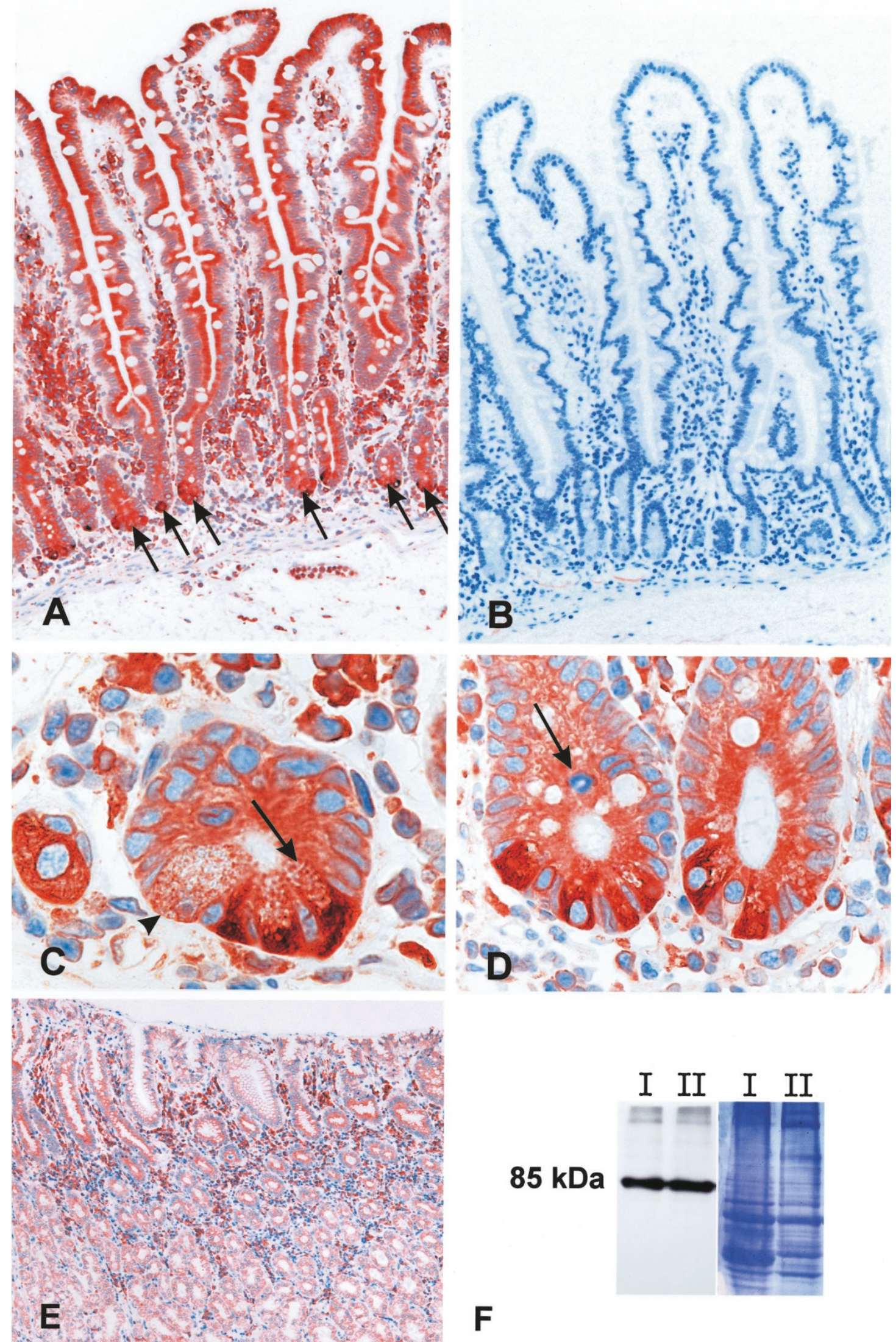

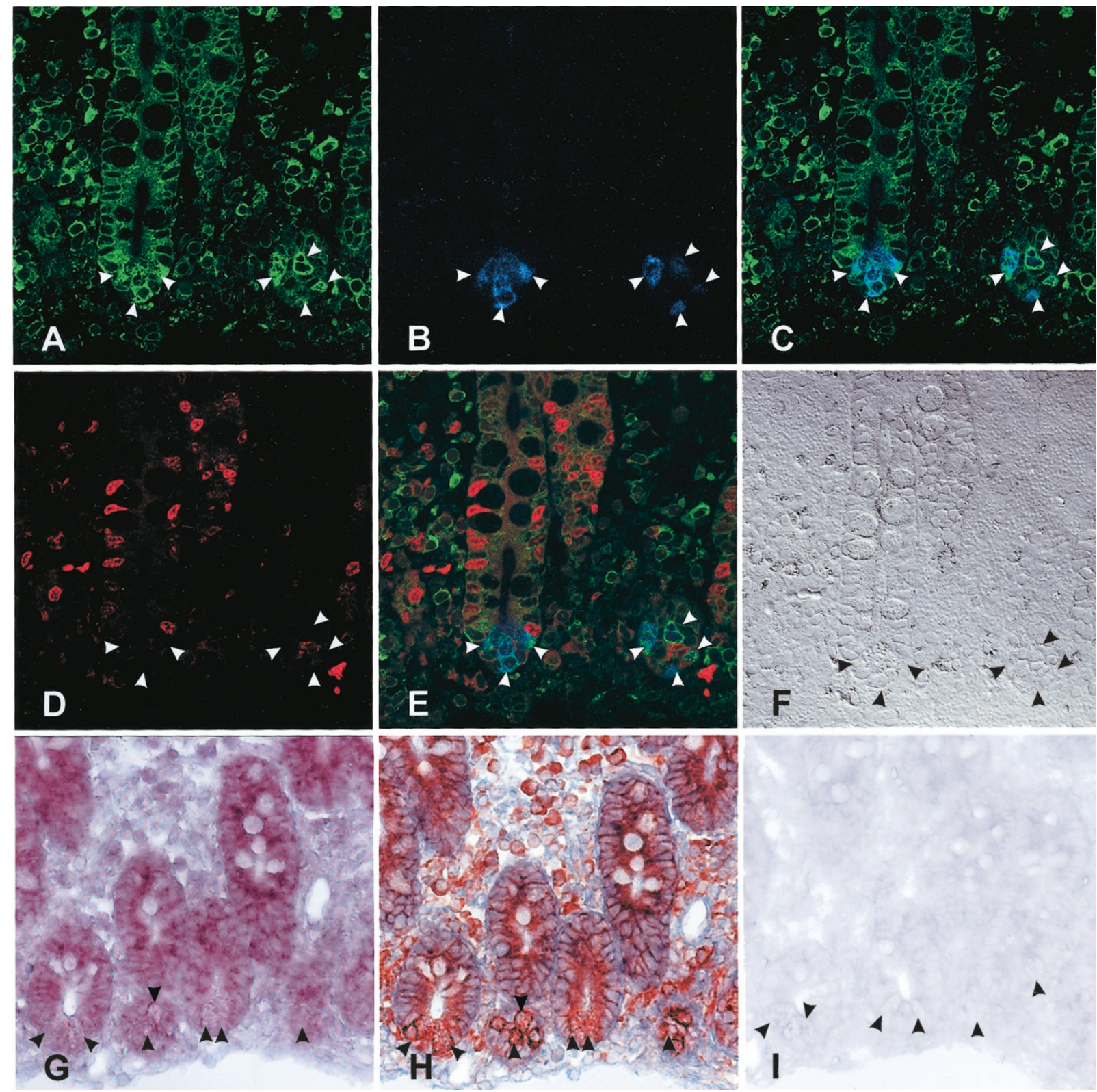

\section{Figure 3.}

Immunofluorescence of a deparaffinized section of normal small intestinal tissue after immunostaining against $\mathrm{Cnx}$ ( $\mathrm{A}, \mathrm{C}$, and $\mathrm{E}$; green), lysozyme (B, C, and E; blue), and Ki67 (D and E; red); and serial sections of normal small intestinal tissue after mRNA in situ hybridization ( $\mathrm{G}$ to $\mathrm{I})$ and DAB immunostaining against Cnx (H). A, In the surface epithelium, strong immunofluorescence of the $\mathrm{Cnx}$ protein (green) is found in isolated cells at crypts of Lieberkühn (arrowheads). B, These cells are identified as Paneth cells (arrowheads) by cosynthesis of lysozyme (blue; see overlay in C). D, Nuclear Ki67 protein (red) is found in several epithelial cells; however, Paneth cells (arrowheads) remain unstained. E, The triple immunofluorescence demonstrates the strong synthesis of Cnx (green) in Paneth cells (arrowheads) characterized by lysozyme (blue) versus the missing synthesis of Ki67 in Paneth cells of normal small intestine. F, Interference phase contrast view of the same section (arrowheads indicate Paneth cells). G, In situ hybridization for mRNA using digoxigenin-labeled antisense riboprobes to Cnx (arrowheads indicate Paneth cells). H, DAB immunostaining against $\mathrm{Cnx}$ on the same tissue section used for mRNA in situ hybridization as shown in G reveals a strong synthesis of the $\mathrm{Cnx}$ protein in Paneth cells (arrowheads). I, Serial tissue section from $\mathrm{G}$ and $\mathrm{H}$ after mRNA in situ hybridization using digoxigenin-labeled sense riboprobes to $\mathrm{Cnx}$ (control; arrowheads indicate Paneth cells). Original magnification: A to $\mathrm{F}, \sim \times 40 ; \mathrm{G}$ to $\mathrm{I}, \sim \times 40$.

\section{Figure 2.}

Section of paraffin-embedded normal small intestinal tissue after diaminobenzidine (DAB) immunostaining against Cnx. A, Strong immunostaining of Cnx is found in Paneth cells at crypts (arrows) and enterocytes at villi. In the interstitium, Cnx is found in mononuclear cells. B, Similarly processed section as shown in A, in which the anti-Cnx antibody had been totally omitted and replaced by the appropriate immunoglobulin fraction (negative control). C, Crypt of Lieberkühn with four Paneth cells clearly identified by the foamy appearance of secretory granules (arrow). The immunostaining of Cnx is reduced when the density of secretory granules increased (compare the cell indicated by an arrow with the cell indicated by the arrowhead). In the Paneth cell indicated by the arrowhead, nuclear shrinkage/karyorrhexis is paralleled by high density of secretory granules, whereas the immunostaining of $\mathrm{Cnx}$ is significantly reduced. D, Crypts of Lieberkühn with immunoreactive Cnx in a mitotic stem cell (arrow) close to Paneth cells with strong $\mathrm{Cnx}$ synthesis. E, Immunostaining of Cnx in normal gastric fundic mucosa. Interstitial mononuclear cells and epithelial cells of gastric glands synthesized the $\mathrm{Cnx}$ protein. $\mathrm{F}$, Western blot analysis of $\mathrm{Cnx}$ protein ( $85 \mathrm{kDa}$ ) synthesis in normal gastric mucosa ( $I$ ) and in normal small intestine $(I /)$ on the left. On the right, the Coomassie-stained polyvinylidene difluoride membrane is shown. Original magnification: $A$ and $B$, $\sim \times 20$; $C$ to $E$, $\sim \times 60$. 
Table 1. Frequency of different Paneth cell immunophenotypes in normal small intestine, complete intestinal metaplasia, and Paneth cell-rich adenomas

\begin{tabular}{|c|c|c|c|c|c|}
\hline \multirow[b]{2}{*}{ Tissue } & \multirow{2}{*}{$\begin{array}{l}\text { Frequency of Paneth } \\
\text { cells per tissue } \\
\text { section } \\
\text { (immunophenotype) }\end{array}$} & \multicolumn{4}{|c|}{ Expression of: } \\
\hline & & Cnx protein & Lysozyme protein & Ki67 protein & Cnx mRNA \\
\hline \multirow[t]{2}{*}{ Normal small intestine } & $55-79 \%$ & +++ & + & - & + \\
\hline & $21-45 \%$ & + & +++ & - & + \\
\hline \multirow{3}{*}{$\begin{array}{l}\text { Complete intestinal } \\
\text { metaplasia }\end{array}$} & $35-70 \%$ & +++ & + & - & + \\
\hline & $27-45 \%$ & + & ++ & - & + \\
\hline & $3-20 \%$ & + & + & + & + \\
\hline $\begin{array}{l}\text { Paneth cell-rich } \\
\text { adenoma }\end{array}$ & $100 \%$ & + & $+/-$ & + & + \\
\hline
\end{tabular}

In normal small intestine, two immunophenotypes were found, characterized by the inverse staining intensity of Cnx and lysozyme. In complete intestinal metaplasia, one further immunophenotype of Paneth cells was found, characterized by nuclear Ki67 protein and very low immunostaining of Cnx and lysozyme. This immunophenotype was exclusively found in Paneth cells of Paneth cell-rich adenomas.

of Lieberkühn (Bry et al, 1994). With regard to Paneth cell morphology and synthesis/storage of secretory granules (lysozyme), the characteristic Cnx immunostaining reflects different stages of Paneth cell differentiation and function. However, the variation in $\mathrm{Cnx}$ and lysozyme immunostaining and the differences in cellular morphology indicate that Paneth cells of different cellular activity/life stage exist together in a single crypt of the normal human small intestine.

Concerning intestinal metaplasia, histopathologic and histochemical techniques allow the identification of different types: (i) type I, which is characterized by the presence of Paneth cells, absorptive cells, and goblet cells secreting sialomucins and which corresponds to the small intestine phenotype (complete intestinal metaplasia); (ii) type II, which is characterized by the presence of columnar and goblet cells secreting sialomucins; and (iii) type III, which is characterized by the presence of columnar and goblet cells secreting sulfomucins (Rouzier and Robine, 2001). Paneth cells are not found in type II and type III intestinal metaplasia; therefore the lesions have been designated as incomplete intestinal metaplasia. It has been suggested that intestinal metaplasia might be a preneoplastic hyperproliferative lesion of the stomach (Cahill et al, 1996; Correa, 1988; De Bolos et al, 2001; Dixon, 2001; Endoh et al, 1999; Morson, 1955; Rokkas et al, 1991; Rouzier and Robine, 2001; Wong et al, 2000; Tahara, 1995). However, cellular differentiation and proliferative status of Paneth cells in complete intestinal metaplasia (type I metaplasia) of the stomach has not been extensively examined. Our findings that the expression of $\mathrm{Cnx}$ by Paneth cells is reduced in complete intestinal metaplasia of the stomach and that nuclear expression of the Ki67 protein is found in such Paneth cells argue for a dysregulated cell cycle of Paneth cells in complete intestinal metaplasia. These results are in agreement with data recorded by Wong et al (2000). However, further studies are necessary to elucidate whether complete intestinal metaplasia constitutes a precancerous lesion in itself or provides a milieu conductive to the development of gastric cancer.

In Paneth cell-rich adenomas, Cnx was significantly reduced and accompanied by an even higher proliferative activity (Ki67 expression) when compared with complete intestinal metaplasia. These findings indicate a misdirected cellular differentiation of Paneth cells, which seems to be more prominent in Paneth cell-rich adenomas than in complete intestinal metaplasia. Although both conditions are considered as preneoplastic hyperproliferative lesions, it is surprising that tumors derived from Paneth cells are not frequent and that the entity of Paneth cell-carcinoma has not been established yet (Endoh et al, 1999; Heitz and Wegmann, 1980; Nevalainen et al, 1998; Rubio et al, 1996). However, it should be taken into consideration that Paneth cell-rich carcinomas might derive from dysregulated stem cells rather than from formerly well-differentiated Paneth cells.

Expression and distribution of the $\mathrm{Cnx}$ protein in mitosis were further investigated by cell culture experiments. Because primary cultures of differentiated human Paneth cells are not yet well established, the intestinal epithelial cell line $\mathrm{CaCo} 2$ was used (Cario et al, 1999; Jumarie and Malo, 1991). In mitotic CaCo2 cells, immunoreactivity to $\mathrm{Cnx}$ was found in numerous dispersed cisternae and vesicles, a common phenomenon observed for proteins associated with the ER (Jesch and Linstedt, 1998). The strong immunostaining for $\mathrm{Cnx}$ in such metaphase cells is obviously a result of a condensation of the cytoplasm, including the ER vesicles. Western blot analyses gave evidence that the total amount of immunoreactive $\mathrm{Cnx}$ was not affected by mitosis itself. Although the mechanisms which drive the mitotic cisternal tubulation and condensation are currently unknown, some authors have proposed that these effects result from an imbalance in membrane traffic on the secretory pathway of mitotic cells (Farmaki et al, 1999).

In this study, the distribution of $\mathrm{Cnx}$ protein was generally corroborated by the results of mRNA in situ 

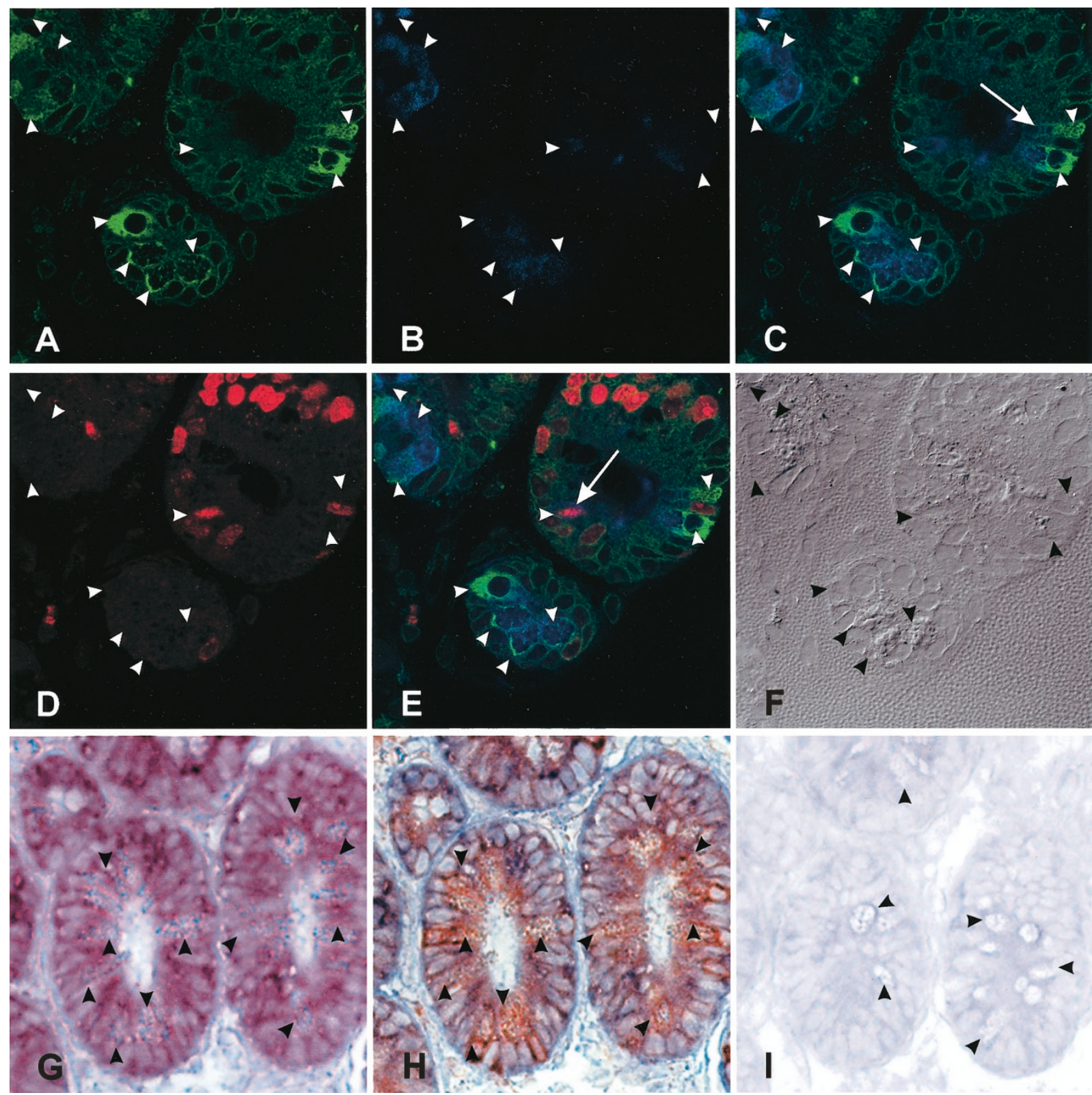

\section{Figure 4.}

Immunofluorescence of a deparaffinized section of complete intestinal metaplasia of the stomach after immunostaining against $\mathrm{Cnx}(\mathrm{A}, \mathrm{C}$, and $\mathrm{E}$; green), lysozyme (B, C, and E; blue), and Ki67 ( $D$ and E; red); and serial sections of complete intestinal metaplasia after mRNA in situ hybridization ( $\mathrm{G}$ to I) and DAB immunostaining against $\mathrm{Cnx}(\mathrm{H})$. A, Strong immunofluorescence of the $\mathrm{Cnx}$ protein (green) is found in a small number of Paneth cells identified by cosynthesis of lysozyme (blue; B) (arrowheads indicate Paneth cells). C, The overlay of $\mathrm{Cnx}$ (green) and lysozyme (blue) shows that in Paneth cells (arrowheads), a moderate immunostaining against Cnx is not regularly associated with a high density of lysozyme (arrow). D, Nuclear Ki67 protein (red) is found in several proliferating cells (arrowheads indicate Paneth cells). E, Triple immunofluorescence of lysozyme (blue), nuclear Ki67 (red), and Cnx (green) in a Paneth cell (arrow) of intestinal metaplasia. The majority of Ki67-producing cells (red) do not show any staining for lysozyme (blue), indicative for proliferating enterocytes (arrowheads indicate Paneth cells). F, Interference phase contrast view of the same section (arrowheads indicate Paneth cells). G, In situ hybridization for Cnx mRNA using digoxigenin-labeled antisense riboprobes. Cnx mRNA is found in Paneth cells (arrowheads). H, The same tissue section used for mRNA in situ hybridization (G) with DAB immunostaining against Cnx (arrowheads indicate Paneth cells). Immunostaining of $\mathrm{Cnx}$ is found in Paneth cells (arrowheads). I, Serial tissue section from $\mathrm{G}$ and $\mathrm{H}$ after mRNA in situ hybridization using digoxigenin-labeled sense riboprobes to $\mathrm{Cnx}$ (control; arrowheads indicate Paneth cells). Original magnification: A to F, $\sim \times 60$; G to I, $\sim \times 60$.

hybridization experiments. Nevertheless, true quantification of $\mathrm{Cnx}$ is not provided by these methods, and immunohistochemical data must be substantiated by other techniques, such as Western blot analysis, ELISA, or qRT-PCR. Unfortunately, the application of all these methods was limited by the use of paraffinembedded formalin-fixed tissues. Because fresh or frozen tissue specimens of Paneth cell metaplasia and
Paneth cell-rich adenoma were not available, we were not able to systematically address the phenomenon of $\mathrm{Cnx}$ in Paneth cell metaplasia and the true relation of $\mathrm{Cnx}$ to Paneth cell differentiation and function could be underestimated.

In summary, expression and synthesis of $\mathrm{Cnx}$ by Paneth cells probably reflects certain stages of Paneth 

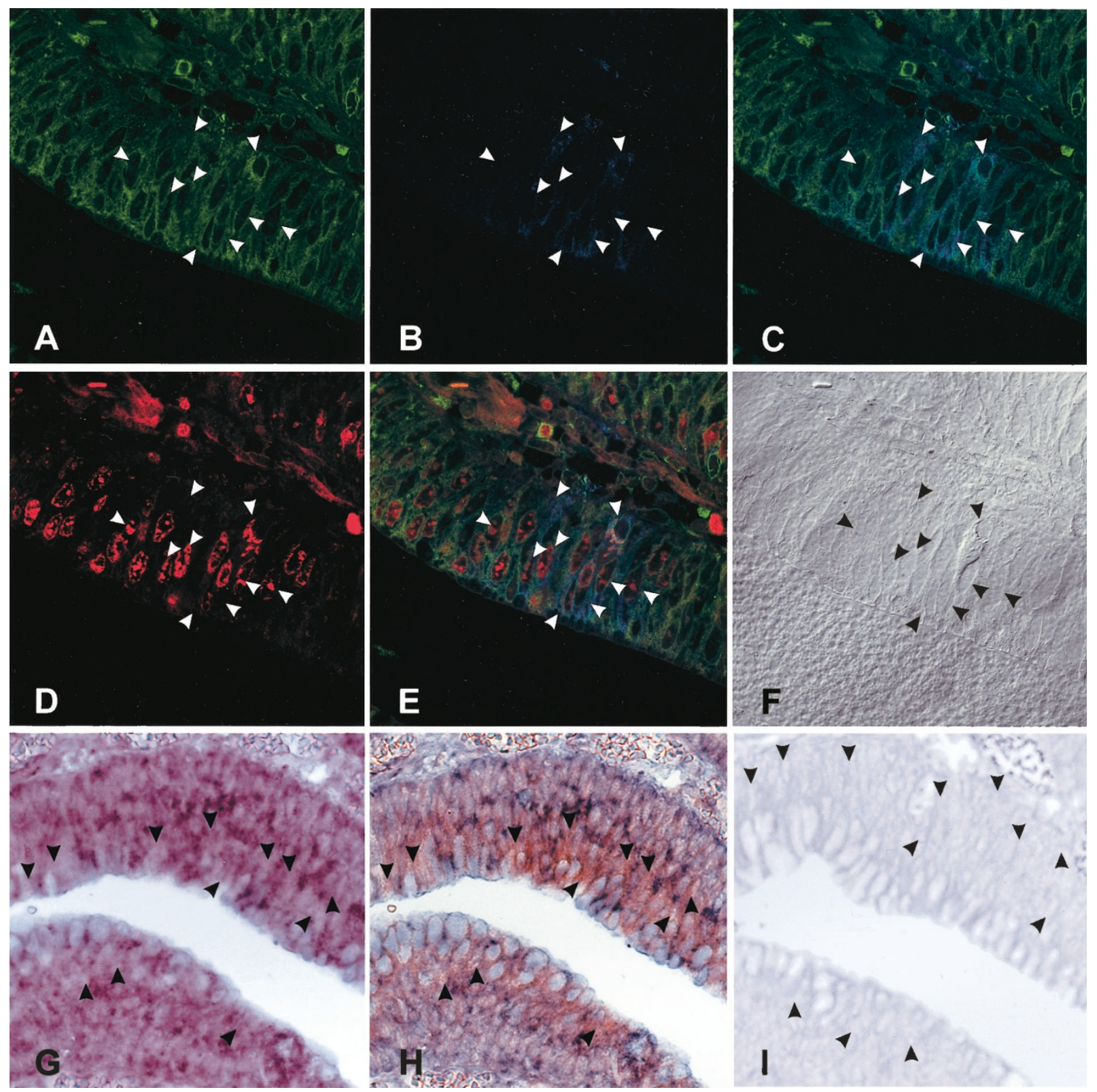

\section{Figure 5.}

Immunofluorescence of a deparaffinized section of Paneth cell-rich adenoma after immunostaining against $\mathrm{Cnx}$ (A, C, and E; green), lysozyme (B, C, and E; blue), and Ki67 (D and E; red); and serial sections of complete intestinal metaplasia after mRNA in situ hybridization ( $G$ to $\mathrm{I}$ ) and DAB immunostaining against Cnx (H). A, Homogeneous moderate immunostaining against $\mathrm{Cnx}$ (green) is found (arrowheads indicate Paneth cells). B, Paneth cells indicated by arrowheads are identified by production of lysozyme (blue). A and B are merged in C (arrowheads indicate Paneth cells). D, Nuclear Ki67 protein (red) is frequently found (arrowheads indicate Paneth cells). E, In Paneth cell-rich adenomas, triple immunofluorescence of nuclear Ki67 (red), lysozyme (blue), and Cnx (green) demonstrates a high number of proliferating Paneth cells (arrowheads). F, Interference phase contrast view of the same section (arrowheads indicate Paneth cells). G, In situ hybridization for mRNA using digoxigenin-labeled antisense riboprobes to $\mathrm{Cnx}$. Cnx mRNA is diffusely distributed among the epithelial cells (arrowheads indicate Paneth cells). H, The same tissue section used for mRNA in situ hybridization (G) with DAB immunostaining against Cnx reveals a moderate immunostaining (arrowheads indicate Paneth cells) I, Serial tissue section from $\mathrm{G}$ and $\mathrm{H}$ after mRNA in situ hybridization using digoxigenin-labeled sense riboprobes to $\mathrm{Cnx}$ (control; arrowheads indicate Paneth cells). Original magnification: A to $\mathrm{F}, \sim \times 60 ; \mathrm{G}$ to $\mathrm{I}, \sim \times 60$.

cell differentiation and function. Strong immunostaining of the Cnx protein is associated with an early stage of Paneth cell secretory function. In later stages, the intensity of $\mathrm{Cnx}$ increasingly declines, with an increase in the density of secretory granules (accumulation of lysozyme) or nuclear shrinkage/karyorrhexis. In Paneth cell-rich adenomas and in complete intestinal metaplasia, a decline of $\mathrm{Cnx}$ immunoreactivity is apparently associated with expression of nuclear Ki67 protein, indicating cell cycle dysregulation of Paneth cells. Our results do not necessarily indicate that $\mathrm{Cnx}$ has a function in cellular proliferation.

\section{Materials and Methods}

\section{Patients}

A total of 15 small intestinal samples ( 6 men, 9 women; mean age, 51 years; range, 32 to 62 years), 35 gastric 

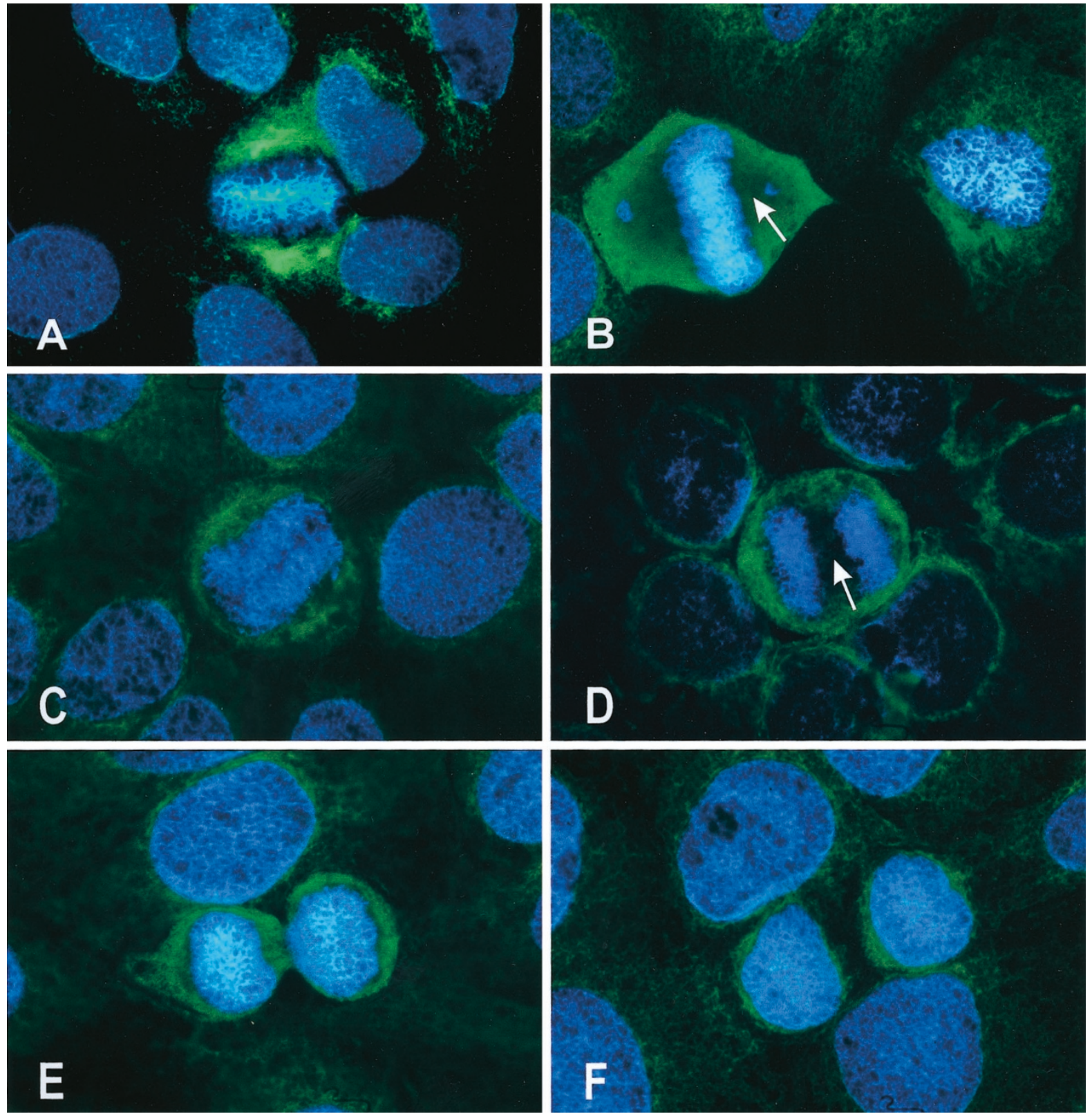

\section{Figure 6.}

$\mathrm{CaC} 02$ cells in different stages of mitosis with immunostaining of $\mathrm{Cnx}$ (green) and nuclear staining with Hoechst 33258 (blue). In interphase CaCo2 cells, the Cnx protein is distributed in a fine reticulum filling the cytoplasm, concentrated near the nuclear membrane. Condensation of the Cnx protein is found in different stages of mitosis: A, prometaphase; B, metaphase; C, early anaphase; D, late anaphase; E, early telophase; and F, late telophase. Note, the anti-Cnx staining is reduced in the mitotic spindle apparatus (arrow in B), and the staining is not found between the drifting chromosomes (arrow in D). The pictures shown are representative for a high number of cells examined at the different stages of cellular division. Original magnification: A to $\mathrm{F}, \sim \times 60$.

samples with complete intestinal metaplasia $(11$ men, 24 women; mean age, 63 years; range, 48 to 83 years), and 5 cases of Paneth cell-rich adenoma of the colon/rectum (3 men, 2 women; mean age, 65 years; range, 51 to 75 years) were included in the study. All tissues were obtained by biopsy. For molecular procedures, normal gastric and small intestinal tissues were used from surgical resections for gastric cancer or cancer of the ascending colon (1 men, 4 women; mean age, 61 years; range, 33 to 80 years). Mucosal tissue layers were mechanically dissected from the underlying submucosa, immediately cooled in liquid nitrogen, and stored at $-80^{\circ} \mathrm{C}$ until use. All diagnoses were established independently by conventional histologic criteria on hematoxylin and eosin-stained sections of paraffin-embedded tissues by two authors (FA and NG). The use of human tissues was approved by Heidelberg University.

\section{Production of $m A b$}

Antibody was obtained by fusing immune B cells from the spleen of a boost primed Sprague-Dawley rat (Janvier, Le Geneste-St Isle, France) with P3X63Ag8 


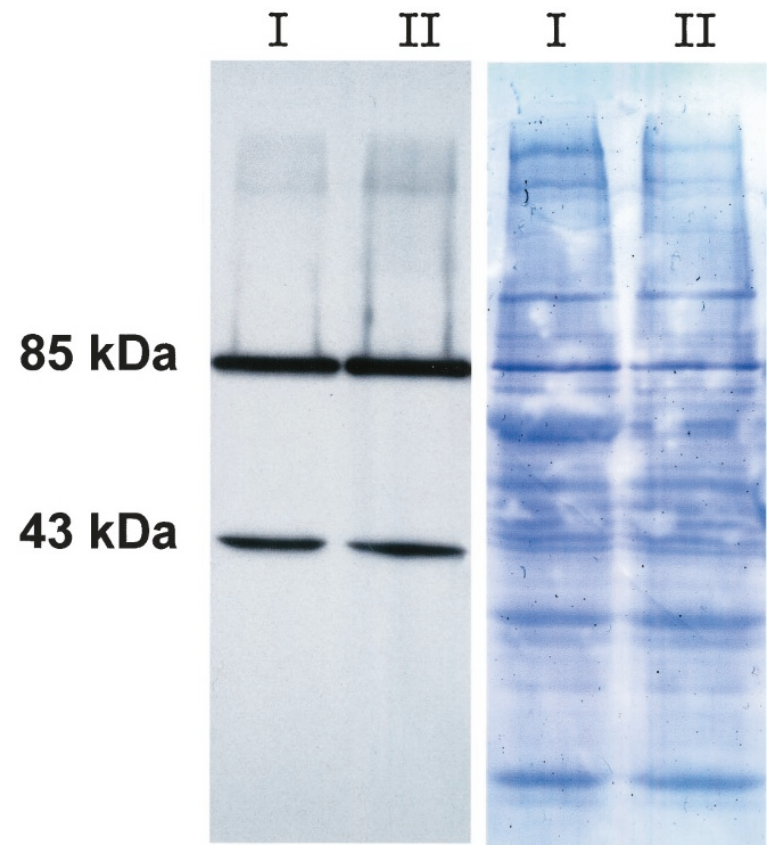

Figure 7.

Western blot analysis of $\mathrm{Cnx}$ protein synthesis in $\mathrm{CaC} 02$ cells treated with thrombin-activated FXIII (I) and in untreated CaCo2 cells (II) after 60 hours of incubation (panel). On the right, the Coomassie-stained polyvinylidene difluoride membrane is shown. The analyzed homogenates yielded comparable $\mathrm{Cnx}$ protein amounts $(85 \mathrm{kDa})$. $\beta$-actin labeling $(43 \mathrm{kDa})$ is shown as loading control.

tumor cells from a BALB/c mouse (CRL-1597; American Type Culture Collection [ATCC], Manassas, Virginia) to produce hybridomas, following the classic protocols (Yokoyama, 1991). Briefly, immunization of the 8-week-old rat was performed using a liquid preparation of mature $\mathrm{Cnx}$ protein in $0.9 \% \mathrm{NaCl}$ from normal purified human small intestinal crypts (Grossmann et al, 1998) of a 55-year-old female. First and second immunizations were performed subcutaneously in the presence of Specol adjuvants (ID-DLO, Lelystad, Netherlands). The antigen was additionally applied in five booster immunizations intravenously, without Specol, over 70 days. Fusion of harvested spleen cells and tumor (myeloma) cells (ratio 5:1) was performed at $37^{\circ} \mathrm{C}$ with application of polyethylene glycol (Sigma, Deisenhofen, Germany). The resulting pellet was harvested and plated into tissue culture plates (Greiner, Frickenhausen, Germany) with RPMI 1640 (Gibco Invitrogen Corporation, New York)/15\% FCS (Sigma)/HAT (hypoxanthine, azaserine, and thymidine) medium. On day 15, HAT was replaced by HT (both substances purchased from Sigma), and the RPMI 1640/15\% FCS/HT medium was used for a further 5 days before a standard RPMI 1640/10\% FCS medium was applied for screening experiments. The supernatants were tested in a first screening assay for the presence of the desired antibody with a reticular cytoplasmic staining: cryosections of human intestine were incubated with undiluted supernatants for 1 hour and then visualized by a Cy-2-labeled anti-rat IgG antibody (Rockland, Gilbertsville, Pennsylvania). Candidate hybridomas identified were subcloned and subsequently tested. The hybridoma cell line BB1F10 was established, when stable clones were identified, frozen in liquid nitrogen, and successfully thawed. The mAb was purified by antibody affinity chromatography using a HiTrap Protein G HP column following the manufacturer's instructions (Amersham Pharmacia Biotech, Little Chalfont, England) and further characterized by isotype-specific mapping using a Western blot technique (see below).

\section{cDNA Library and Immunoscreening}

A sample of purified 2- $\mu \mathrm{g}$ mRNA from human intestinal mucosa was used for cDNA synthesis and construction of a custom library in $\lambda$ TriplEx (Promega, Madison, Wisconsin). Approximately $2.4 \times 10^{6}$ plaques were plated, transferred to nitrocellulose filters (Schleicher \& Schuell, Dassel, Germany) treated with isopropylthiogalactoside (Sigma), and subsequently incubated with the purified mAb overnight (dilution 1:2500 in 5\% skim milk TBST). For further detection a secondary horseradish peroxidase (HRP)labeled antibody against rat IgG (1:5,000; Santa Cruz Biotechnology, Santa Cruz, California) and the ECL substrate (Amersham Pharmacia Biotech) were used.

\section{Analysis of Inserts}

Conversion of $\lambda$ TriplEx in pTriplEx was performed following the manufacturer's protocol (Clontech, Palo Alto, California). The inserts were PCR amplified (4 minutes of denaturation at $94^{\circ} \mathrm{C}, 30$ cycles of denaturation at $94^{\circ} \mathrm{C}$ for 60 seconds, annealing at $55^{\circ} \mathrm{C}$ for 60 seconds, and extension at $72^{\circ} \mathrm{C}$ for 60 seconds) using a set of $\lambda$ TriplEx screening amplimers (Clontech). PCR products were sequenced by an automated sequencing system ( $\mathrm{ABI}$, Weiterstadt, Germany). All sequencing reactions were performed with 100 ng of each purified PCR product and $10 \mathrm{pmol}$ of the appropriate primer (see above) using the Big Dye Terminator kit (ABI). Reactions were run on an $A B I$ 3700 capillary sequencer according to the manufacturer's recommendations. Sequences were assembled using the program SeqMan (Lasergene, Madison, Wisconsin), and GenBank searches were performed.

\section{Protein G Bead-mAb Column and Immunoaffinity Chromatography}

The affinity-purified mAb (approximately $13 \mathrm{mg} / \mathrm{ml}$ ) was covalently bound to protein G Sepharose (Amersham Pharmacia Biotech) using dimethylpimelimidate-dihydrochloride (Sigma). The matrix was disposed in a 5-ml column (Pierce, Rockford, Illinois). Immunoaffinity chromatography was performed according to the manufacturer's recommendations. Briefly, total protein was isolated from human normal intestine as previously described (Gassler et al, 2001). After an incubation period of 24 hours, the protein lysate was removed and the column was washed twice in PBS. Elution of the antigen was achieved by application of $1 \mathrm{ml}$ of $0.1 \mathrm{M}$ glycine, $\mathrm{pH}$ 2.6. The samples were purified by SDS-PAGE $(7.5 \%)$ and fur- 
ther analyzed by Western blot technique and MALDI mass spectrometry (for details see below).

\section{MALDI Mass Spectrometry}

The protein band $(85 \mathrm{kDa})$ was excised from the $7.5 \%$ gel (see below). The gel pieces were washed repeatedly and tryptic digestion was performed at $37^{\circ} \mathrm{C}$ overnight using sequencing grade modified trypsin (Promega) in $40 \mathrm{~mm}$ ammonium bicarbonate. The reaction was stopped by freezing. MALDI mass spectra were recorded in the positive ion reflector mode with delayed extraction on a Reflex II time-of-flight instrument (Bruker-Daltonik GmbH, Bremen, Germany) equipped with a SCOUT multiprobe inlet and a 337-nm nitrogen laser. Ion acceleration voltage was set to $20.0 \mathrm{kV}$, the reflector voltage was set to $21.5 \mathrm{kV}$, and the first extraction plate was set to $15.4 \mathrm{kV}$. Mass spectra were obtained by averaging 50 to 200 individual laser shots. Calibration of the spectra was performed internally by a two-point linear fit using the autolysis products of trypsin at $\mathrm{m} / \mathrm{z} 842.50$ and $\mathrm{m} / \mathrm{z}$ 2211.10.

Sample preparation for post-source decay (PSD) analysis was achieved by cocrystallization of matrix (alpha-cyano-4-hydroxycinnamic acid saturated in 50\% acetonitrile/water) with ZipTip C18 (Millipore, Bedford, Massachusetts) concentrated samples as previously described (Regula et al, 2000). PSD analysis was performed in the positive ion reflector mode with delayed extraction by setting an ion gate width of $40 \mathrm{Da}$ around the ion of interest. Data were acquired in 14 segments by decreasing the reflector voltage in a stepwise fashion. For each segment 100 to 200 individual laser shots were accumulated. The fragment ion spectrum was obtained by pasting together all segments to a single spectrum using the FAST software provided by Bruker-Daltonik. Fragment ion calibration was performed externally with the fragment masses of the adrenocorticotropic hormone 18-39 clip.

Singly charged monoisotopic peptide masses were used as inputs for database searching. Searches were performed against the NCBInr database using the ProFound search algorithm (http://129.85.19.192/ prowl-cgi/ProFound.exe) and the protein prospector software developed at the University of California, San Francisco (http://prospector.ucsf.edu). Mass tolerance for the monoisotopic peptide masses was set to $\pm 100 \mathrm{ppm}$ or $\pm 0.1 \mathrm{Da}$. Searches with fragment masses from PSD experiments were performed against the NCBInr database using the MS-Tag search algorithm provided by the protein prospector software package. Parent mass tolerance was set to $\pm 0.1 \mathrm{Da}$, and fragment ion tolerance was set to \pm 0.7 $\mathrm{Da}$.

\section{Cell Culture}

CaCo2 cells (HTB-37; ATCC) were routinely cultured in DMEM (Gibco Invitrogen) containing 10\% FCS (Sigma). All experiments were performed with cells between passages 50 and 65 . Recombinant human
FXIII, consisting of two $\alpha$-chains ( $\alpha_{2}$ configuration), and thrombin $(1,000 \mathrm{IU} / \mathrm{ml})$ were kindly provided by the Centeon $\mathrm{GmbH}$ (Marburg, Germany).

\section{Cell Proliferation Assay}

The assay was performed following the protocol of Cario et al (1999). Briefly, approximately 3 to $5 \times 10^{4}$ $\mathrm{CaCo} 2$ cells were seeded per $94-\mathrm{mm}$ plate (Greiner) and cultured for the first 24 hours in DMEM containing $10 \%$ FCS. Then the cells were cultured in DMEM without FCS for a further 48 hours; all proliferation experiments were performed in DMEM completely free of FCS. For immunofluorescence experiments, $\mathrm{CaCo} 2$ cells were seeded on coverslips. Proliferation of $\mathrm{CaCo} 2$ cells was induced by thrombin-activated FXIII [activation of FXIII with thrombin at a ratio $3: 1$ ]. After 24,48 , and 60 hours of incubation at $37^{\circ} \mathrm{C}$ and $5 \% \mathrm{CO}_{2}$, the cells were analyzed by both Western blot and immunofluorescence (see below). For Western blot analysis, approximately $5 \times 10^{4}$ cells per group were homogenized in $3 \mathrm{ml}$ of TriReagent (Sigma) on ice using the Ultra Turrax equipment (IKA Labortechnik, Staufen, Germany).

\section{SDS-PAGE and Western Blot Analysis}

Proteins resolved by one-dimensional SDS-PAGE (7.5\%; approximately $15 \mu \mathrm{g}$ of total protein loaded per lane) were transferred to an Immobilon-P (Millipore) polyvinylidene difluoride membrane using a semi-dry blot system. Molecular weight markers were purchased from Amersham Pharmacia Biotech. For immunoreaction, the anti-Cnx mAb $(1: 1,000)$, the secondary HRP-conjugated anti-rat antibody [1:10,000; Santa Cruz), and the ECL substrate (Amersham Pharmacia Biotech) were applied as previously described (Gassler et al, 2001). For isotype-specific mapping, the following secondary antibodies were used: mouse anti-rat IgG1, mouse anti-rat lgG2a, and mouse antirat IgG2b (all antibodies from Serotec, Oxford, United Kingdom). The amount of each sample analyzed was additionally adjusted to the intensity of $\beta$-actin staining. Therefore, blots were stripped of antibodies and reprobed with an anti- $\beta$-actin antibody $(0.4 \mu \mathrm{g} / \mathrm{ml}$; Sigma) and a secondary HRP-conjugated anti-mouse antibody (1:10,000; Santa Cruz). For antibody stripping, blots were immersed in pure methanol, washed in water, and incubated in stripping buffer consisting of $2 \%$ SDS, $100 \mathrm{~mm} \beta$-mercaptoethanol, and $62.5 \mathrm{~mm}$ Tris- $\mathrm{HCl}(\mathrm{pH} 7.5)$ for 30 minutes at $50^{\circ} \mathrm{C}$. Subsequently, blots were processed as described. Negative controls included similarly processed blots in which the primary antibody was totally omitted.

\section{Immunohistochemistry/Immunofluorescence/Data Analysis}

Paraffin-embedded tissues were sectioned (2 $\mu \mathrm{m})$, mounted on pretreated slides (DAKO, Glostrup, Denmark), air dried overnight, and subsequently deparaffinized. After microwave treatment (citrate buffer; 500 $\mathrm{W}, 10$ minutes), the anti-Cnx antibody (1:200 in PBS) 
was applied and tissues were incubated for 1 hour at room temperature in a moist chamber. In additional experiments on serial sections, a mouse antichromogranin mAb (1:10; Linaris, WertheimBettingen, Germany) was applied. Antibody labeling was visualized using the routine $A B C$ technique and diaminobenzidine as a chromogen following the manufacturer's recommendations (DAKO).

For triple-labeling experiments, sections were incubated for 60 minutes with a mixture of all primary antibodies (rat anti-Cnx [10 $\mu \mathrm{g} / \mathrm{ml}]$; rabbit anti-lysozyme/muramidase [50 $\mu \mathrm{g} / \mathrm{ml}$; DAKO]; mouse antiKi67/Mib-1 [70 $\mu \mathrm{g} / \mathrm{ml}$; Dianova, Hamburg, Germany]) and afterward detected with the appropriate secondary antibodies (Cy2 anti-rat [1:200; Rockland]; AMCA anti-rabbit [1:20; Dianova]; tetramethylrhodamine isothiocyanate anti-mouse [1:30; Dianova]). Each incubation time was followed by three 3-minute washes with PBS. Histosafe-mounted sections were viewed with an ultraviolet laser scanning microscope (LSM 510; Zeiss, Jena, Germany). For simultaneous immunofluorescence, different laser systems were used (346, 488, and $543 \mathrm{~nm}$ ) with the corresponding barrier filters. Nuclear staining of cultured $\mathrm{CaCo} 2$ cells was performed with Hoechst 33258 (Sigma). Computer images were collected on optical memory discs and arranged with the Corel Draw program. For single immunofluorescence the staining procedure was identical to that detailed above. Negative controls included similarly processed sections in which the primary antibody had been totally omitted or replaced by the appropriate immunoglobulin fraction.

To estimate the number of Paneth cells with different immunophenotypes (triple immunostaining against Cnx, lysozyme, Ki67), five randomly selected microscopic fields were analyzed blindly $(\mathrm{BH})$ per tissue section (one section per patient; see above). The number of Paneth cells was counted manually under fluorescence microscope (Axiophot 2; Zeiss) using a $\times 40$ objective; data are expressed in percent values.

\section{mRNA In Situ Hybridization}

For mRNA in situ hybridization experiments, the $\mathrm{Cnx}$ insert of pTriplEx (see above) was directionally cloned into the EcoRI-Sall side of pBluescript II KS $( \pm)$ phagemid (Stratagene, La Jolla, California). To generate antisense and sense RNA probes for in situ hybridization experiments, the plasmid was restricted with EcoRI or Sall and then transcribed with T3 or T7 RNA polymerase (Roche, Mannheim, Germany), respectively. The in vitro transcription procedure was performed according to the protocol supplied by the manufacturer (Roche). Deparaffinized tissue sections were postfixed in 4\% paraformaldehyde/PBS for 10 minutes and treated with proteinase $\mathrm{K}(8 \mu \mathrm{g} / \mathrm{ml}$ in PBS) (Roche) for 30 minutes at $37^{\circ} \mathrm{C}$. To reduce background, slides were washed in PBS and acetylated for 15 minutes in 0.1. $\mathrm{m}$ triethanolamine, $\mathrm{pH}$ 8.0, containing $0.25 \%$ acetic anhydride. To improve the penetration of the probes during the in situ hybridization experiments, the transcripts were shortened to a calculated average length of 250 bases by alkaline hydrolysis. Hybridization with a probe concentration of 2 to 4 nanogram probe per microliter of hybridization mixture was performed overnight at a temperature of $46^{\circ} \mathrm{C}$. To demonstrate the specificity of the probe, control experiments were performed with sense probes on alternate tissue sections. Negative controls included tissue sections in which the antisense/sense probe or the anti-digoxigenin antibody was replaced by PBS.

\section{Acknowledgements}

The authors thank Dr. Herbert Spring (Biomedical Structure Analysis Group, German Cancer Research Center, Heidelberg, Germany) for performing laser scanning images and Dr. Timo Nevalainen (Department of Pathology, University of Turku, Finland) for providing sections of paraffin-embedded tissues of Paneth cell-rich adenoma (K92-8544). The help of Drs. A. Alonso and T. Kempf (German Cancer Research Center) is greatly acknowledged. The authors are grateful to Ms. Jutta Scheuerer for expert technical assistance and to Ms. Ursula Horr and Mr. John Moyers for help in processing the photographs. We acknowledge Dr. Hubert Metzner from the Centeon $\mathrm{GmbH}$ for providing FXIII and thrombin.

\section{References}

Bergeron JJM, Brenner MB, Thomas DY, and Williams DB (1994). Calnexin: A membrane-bound chaperone of the endoplasmic reticulum. TIPS 19:124-128.

Bry L, Falk P, Huttner K, Ouellette A, Midtvedt T, and Gordon Jl (1994). Paneth cell differentiation in the developing intestine of normal and transgenic mice. Proc Natl Acad Sci USA 91:10335-10339.

Cahill RJ, Kilgallen C, Beattie S, Hamilton H, and O'Morain C (1996). Gastric epithelial cell kinetics in the progression from normal mucosa to gastric carcinoma. Gut 38:177-181.

Cario E, Goebell H, and Dignass AU (1999). Factor XIII modulates intestinal epithelial wound healing in vitro. Scand J Gastroenterol 34:485-490.

Chevet E, Cameron PH, Pelletier MF, Thomas DY, and Bergeron JJM (2001). The endoplasmic reticulum: Integration of protein folding, quality control, signaling and degradation. Curr Opin Struct Biol 11:120-124.

Correa P (1988). A human model for gastric carcinogenesis. Cancer Res 48:3554-3560.

De Bolos C, Real FX, and Lopez-Ferrer A (2001). Regulation of mucin and glycoconjugate expression: From normal epithelium to gastric tumors. Front Biosci 6:1256-1263.

Dixon MF (2001). Prospects for intervention in gastric carcinogenesis: Reversibility of gastric atrophy and intestinal metaplasia. Gut 49:2-4.

Endoh Y, Tamura G, Motoyama T, Ajioka Y, and Watanabe H (1999). Well-differentiated adenocarcinoma mimicking complete-type intestinal metaplasia in the stomach. Hum Pathol 30:826-832.

Farmaki T, Ponnambalam S, Prescott AR, Clausen H, Tang BL, Hong W, and Lucocq JM (1999). Forward and retrograde 
trafficking in mitotic animal cells. J Cell Science 112:589600 .

Filipe MI, Muooz N, Matko I, Kato I, Pompe-Kirn V, Jutersek A, Teuchmann S, Benz M, and Prijon T (1994). Intestinal metaplasia types and the risk of gastric cancer: A cohort study in Slovenia. Int J Cancer 57:324-329.

Ganz T (2000). Paneth cells: Guardians of the gut cell hatchery. Nat Immunol 1:99-100.

Gassler N, Rohr C, Schneider A, Kartenbeck J, Bach A, Obermüller N, Otto HF, and Autschbach F (2001). Inflammatory bowel disease is associated with changes of enterocytic junctions. Am J Physiol Gastrointest Liver Physiol 281:G216G228.

Goff JS, Barnett JL, Pelke T, and Appelman HD (1997). Collagenous colitis: Histopathology and clinical course. Am J Gastroenterol 92:57-60.

Grossmann J, Maxson JM, Whitacre CM, Orosz DE, Berger NA, Fiocchi C, and Levine AD (1998). New isolation technique to study apoptosis in human intestinal epithelial cells. Am J Pathol 153:53-62.

Heitz PU and Wegmann W (1980). Identification of neoplastic Paneth cells in an adenocarcinoma of the stomach using lysozyme as a marker, and electron microscopy. Virchows Arch A Pathol Anat Histol 386:107-116.

Helenius A and Aebi M (2001). Intracellular functions of N-linked glycans. Science 291:2364-2369.

Inada $\mathrm{K}$, Tanaka $\mathrm{H}$, Nakanishi $\mathrm{H}$, Tsukamoto $\mathrm{T}$, Ikehara $\mathrm{Y}$, Tatematsu K, Nakamura S, Porter EM, and Tatematsu M (2001). Identification of Paneth cells in pyloric glands associated with gastric and intestinal mixed-type intestinal metaplasia of the human stomach. Virchows Arch 439:14-20.

Jesch SA and Linstedt AD (1998). The Golgi and endoplasmic reticulum remain independent during mitosis in HeLa cells. Mol Biol Cell 9:623-635.

Jumarie C and Malo C (1991). CaCo-2 cells cultured in serum-free medium as a model for the study of enterocytic differentiation in vitro. J Cell Physiol 149:24-33.

Morson BC (1955). Carcinoma arising from areas of intestinal metaplasia in the gastric mucosa. Br J Cancer 9:377-385.

Nevalainen TJ, Haapanen TJ, Rajala P, and Ekfors T (1998). Expression of group II phospholipase $A_{2}$ in Paneth cells of an adenoma of the rectum: A case report. APMIS 106:780-784.

Otto HF (1973). The intestinal Paneth cell: Cytomorphology, ultrastructural pathology and function A contribution on the lysozyme theory. Veroff Morphol Pathol 94:1-75.

Ouellette AJ (1997). Paneth cells and innate immunity in the crypt microenvironment. Gastroentereology 113:1779-1784.

Ouellette AJ (1999). Mucosal immunity and inflammation. IV. Paneth cell antimicrobial peptides and the biology of the mucosal barrier. Am J Physiol (Gastrointest Liver Physiol) 40:G257-G261.

Ouellette AJ and Bevins CL (2001). Paneth cell defensins and innate immunity of the small bowel. Inflamm Bowel Dis $7: 43-50$.
Pai MR, Coimbatore RV, and Naik R (1998). Paneth cell metaplasia in colonic adenocarcinomas. Indian $\mathrm{J}$ Cancer 35:38-41.

Parodi AJ (2000). Protein glucosylation and its role in protein folding. Annu Rev Biochem 69:69-93.

Regula T, Ueberle B, Boguth G, Görg A, Schnölzer M, Herrmann R, and Frank R (2000). Towards a two-dimensional proteome map of Mycoplasma pneumoniae. Electrophoresis 21:3765-3780.

Rokkas T, Filipe MI, and Sladen GE (1991). Detection of an increased incidence of early gastric cancer in patients with intestinal metaplasia type III who are closely followed up. Gut 32:1110-1113.

Rouzier R and Robine S (2001). The subtleties of intestinal metaplasia. Gut 49:8.

Rubio CA, Kanter L, Bjork J, Poppen B, and Bry L (1996). Paneth cell-rich flat adenoma of the rectum: Report of a case. Jpn J Cancer Res 87:109-112.

Shah VI, Ro JY, Amin MB, Mullick S, Nazeer T, and Ayala AG (1998). Histologic variations in the epididymis: Findings in 167 orchiectomy specimens. Am J Surg Pathol 22:990-996.

Stamp GW, Poulsom R, Chung LP, Keshav S, Jeffery RE, Longcroft JA, Pignatelli M, and Wright NA (1992). Lysozyme gene expression in inflammatory bowel disease. Gastroenterology 103:532-538.

Stemmermann GN (1994). Intestinal metaplasia of the stomach: A status report. Cancer 74:556-564.

Tahara E (1995). Molecular biology of gastric cancer. World J Surg 19:484-488.

Vujanic GM and Dojcinov SD (2000). Diversion colitis in children: An iatrogenic appendix vermiformis? Histopathology 36:41-46.

Weaver MG, Abdul-Karim FW, and Srigley JR (1992). Paneth cell-like change of the prostate. Arch Pathol Lab Med 116: 1101-1102.

Wong WM, Stamp GW, Elia G, Poulsom R, and Wright NA (2000). Proliferative populations in intestinal metaplasia: Evidence of deregulation in Paneth and goblet cells, but not endocrine cells. J Pathol 190:107-113.

Yabuki N, Sasano H, Tobita M, Imatani A, Hoshi T, Kato K, Ohara S, Asaki S, Toyota T, and Nagura H (1997). Analysis of cell damage and proliferation in Helicobacter pylori-infected human gastric mucosa from patients with gastric adenocarcinoma. Am J Pathol 151:821-829.

Yokoyama WM (1991). Production of monoclonal antibodies. In: Coligan JE, Kruisbeek AM, Margulies DH, Shevach EM, and Strober W, editors. Current protocols in immunology. New York: John Wiley \& Sons, 2.5.1-2.5.17.

You WC, Blot WJ, Li JY, Chang YS, Jin ML, Kneller R, Zhang L, Han ZX, Zeng XR, Liu WD, Zhao L, Correa P, Fraumeni JF, and Xu GW (1993). Precancerous gastric lesions in a population at high risk of stomach cancer. Cancer Res 53:13171321. 